\title{
Towards Optimization of Open Ended Contact Probes for Breast Cancer Diagnosis
}

\author{
Tuba Yilmaz \\ Department of Electronics and Communication Engineering, Istanbul Technical University, Maslak, \\ Istanbul 34469, Turkey
}

\begin{abstract}
Development of new modalities for breast cancer detection have been a research interest due to the drawbacks of existing diagnostic technologies such as imaging with ionizing waves. In particular, microwave imaging has been investigated in the literature as a new modality for breast cancer detection. Microwave imaging is a promising technique due to the high dielectric contrast between benign and malignant lesions. With this motivation, the dielectric properties of the benign and malignant breast tissues were measured and such measurements have been performed with the open ended contact probes. The open ended contact probe technique widely used in laboratory environment to characterize the dielectric properties of materials with high permittivity and conductivity due to the advantages such as broad band measurement capabilities and limited sample size requirements. The utilization of the open ended contact probe technique as a breast cancer diagnostic technology have been previously envisioned in the literature. One such application is integration of these probes to biopsy guides to diagnose whether the breast lesions are benign or malignant. However, due to the poor measurement accuracy and repeatability the utilization of the technique as a diagnostic technology was not realized. The drawbacks of the technology mostly stem from the mathematical approaches and the deviations from the initial calibration conditions. In this work, to increase the accuracy of the open ended contact probe measurement technique, the probe structure is redesigned by removing the microwave connections and integrating the probe with the RF cable. Probe simulations are performed with Computer Simulation Technology (CST). The probe is tested with both known materials such as methanol and phantom experiments. Oil in gelatine dispersion phantom materials are composed and measured with the designed probe. Probes with small aperture diameters were also evaluated to investigate the potential practical utilization of such probes. Dielectric properties are calculated with an in-house dielectric property retrieval algorithm. A good agreement is obtained with the reference dielectric property measurements.
\end{abstract}

\section{INTRODUCTION}

Open-ended contact probes are widely used for dielectric property measurements of high permittivity and high loss materials, such as biological tissue and sea ice [1]. This measurement technique is preferred due to a number of advantages including but not limited to flexible sample size requirements and broadband measurement capabilities. However, dielectric property measurement with open-ended contact probes suffer from low measurement accuracy and repeatability rates. Therefore, this technique is mostly used in laboratory environment to characterize the dielectric properties of mostly liquid and semi-solid materials.

The developments in microwave imaging and microwave hyperthermia research led to dielectric property characterization of different biological tissues with open-ended contact probes. Dielectric property measurements of all biological tissues are reported in $[2,3,4]$. An extensive study is reported in [5], presenting the dielectric properties of malignant and benign breast tumors. Also, dielectric properties of healthy breast tissues are reported in [6]. Dielectric properties of hepatic malignancies and healthy liver tissue is reported in [7]. The rat liver dielectric properties and rat liver hepatic malignancies are given in [8]. These studies report a significant dielectric property discrepancy between the healthy and malignant tissues. Such discrepancy can enable the use of open ended contact probes as diagnostic equipments. Utilization of open ended contact probes as diagnostic tools was previously envisioned in the literature one example is the use of open-ended contact probes in biopsy guides [9]. Additionally, contact probes also used for dielectric property measurements of other materials such as of gel like tissue mimicking phantoms and liquid phantom materials characterized for testing of microwave sensors and implantable antennas [10, 11, 12].

All reported measurements were performed with open-ended contact probes; although commercially available, dielectric measurements with open-ended contact probes can suffer from high error 
rates. The error mostly stems from the mathematical dielectric property retrieval approach and deviations from the calibration conditions during the measurements. It should be noted that, unlike laboratory environment, the probes should be freely moved during a practical application. For commercial probes the error can increase up to $10 \%$ and it could even be higher when the probe is freely moved during the measurements [13]. Therefore, there is a need to renovate the probe structure to eliminate the error sources.

This work presents an open-ended contact probes fabricated with an integrated cable. The goal is to minimize the error rates by preventing the microwave connections problems that may arise due to cable movements. The open ended contact probes with different aperture sizes are simulated with CST and the probes with $2.2 \mathrm{~mm}$ and $0.9 \mathrm{~mm}$ aperture diameter is fabricated to replicate the commercially available probes and to test the effect of aperture size, respectively. A good agreement is obtained between commercial and fabricated probes. The fabricated probes were also tested with gel-like tissue mimicking phantoms. Aperture dimensions and measurement set-up are given in Section 2. Measurement results are given in Section 3.

\section{METHOD}

\subsection{Simulation and design of the probes}

Probes with different aperture dimensions are simulated and the dielectric properties of the material under test (MUT) is retrieved with an in-house algorithm. Simulated S-parameter response of the probes are used for the dielectric property retrieval of the MUT. In the in-house algorithm the traditional calibration procedure was followed. That is, probes are simulated with deionized water as a reference liquid. Then, short and open simulations were also performed and recorded for calibration. The simulations are performed between $500 \mathrm{MHz}$ to $2 \mathrm{GHz}$ to minimize the computational cost. To simulate the dispersive materials first order Debye model was used. Utilized Debye parameters are given in Table 1. MUT is simulated as a cube where the dimensions of the cube is chosen by calculating the one forth of the wavelength at the lowest frequency of interest. The probe is positioned in the middle of the MUT's top side and immersed $5 \mathrm{~mm}$ inside the MUT. The dimensions of the simulated probes are given in Table 2. The length of the simulated probes are $200 \mathrm{~mm}$. All simulations are performed with Computer Simulation Technology (CST) software.

Table 1: Debye Parameters of the Dispersive Materials

\begin{tabular}{llll}
\hline Material & $\epsilon_{r}$ & $\sigma(S / m)$ & $\tau(s)$ \\
\hline De-ionized Water & 3.1 & 78.4 & $8.27 \mathrm{e}-11$ \\
Methanol & 4.45 & 33.7 & $4.95 \mathrm{e}-11$ \\
\hline
\end{tabular}

Table 2: Aperture Dimensions of the Simulated Probes

\begin{tabular}{llll}
\hline Inner diameter $(\mathrm{mm})$ & Outer diameter $(\mathrm{mm})$ & Dielectric type & $\epsilon_{r}$ \\
\hline 0.8 & 0.24 & PTFE & 2.1 \\
0.9 & 0.28 & PTFE & 2.1 \\
1.5 & 0.44 & PTFE & 2.1 \\
2.2 & 0.65 & PTFE & 2.1 \\
\hline
\end{tabular}

\subsection{Phantom materials}

Phantom materials are frequently employed in the literature to evaluate the performance of the electromagnetic devices designed to operate in biological environment [14]. Both complicated and simple such as, salt and water mixture, phantoms are proposed in the literature [15]. Here ultrawide band oil-in-gelatine dispersion tissue mimicking materials that can mimic the dielectric properties for a wide frequency range is characterized to evaluate the operation of the fabricated probes. A total of three phantoms a wet-skin mimicking phantom, a fat mimicking phantom and a malignant breast tumour mimicking phantom are characterized. Phantom characterization steps are explained in [8]; therefore, it not be detailed in this work. Characterized phantoms were left to solidify overnight. The phantom materials are shown in Fig. 1(a) and Fig 1(b). Recipes for the phantom mimicking materials are shown in Table 3. The phantoms are composed with off-the-shelf materials where the detergents acts as a surfactant that enables the homogeneous mixture of water and oil. Dielectric properties of the detergents are measured and the detergent with lower permittivity is used for the fat phantom. Dielectric property measurements of the detergents are shown in Fig. 2. 


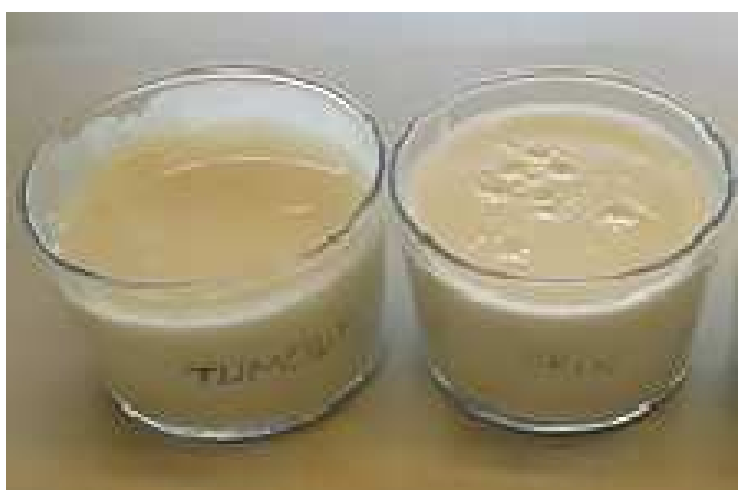

(a)

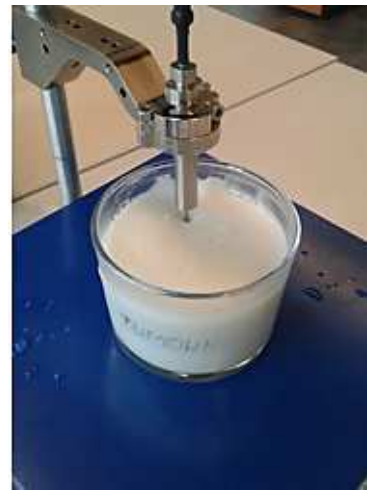

(b)

Figure 1: Oil-in-gelatine dispersion ultrawide band phantom materials (a) Characterized wet-skin and tumour (b) Dielectric property measurement from the phantom material.

Table 3: Recipes for oil-in-gelatine dispersion phantom materials.

\begin{tabular}{llll}
\hline Ingredients & Fat & Wet-skin & Tumour \\
\hline Deionized Water $(\mathrm{gr})$ & 57.4 & 230.0 & 230.0 \\
Gelatine (gr) & 15.0 & 34.1 & 34.1 \\
NaCl (gr) & 0.0 & 1.4 & 1.2 \\
Oil $(\mathrm{gr})_{\text {Detergent }_{1}(\mathrm{gr})}^{329.6}$ & 75.0 & 30.0 \\
Detergent $_{2}(\mathrm{gr})$ & 0.0 & 40.0 & 40.0 \\
\hline
\end{tabular}

\subsection{Measurement set-up}

For measurements, probe with $2.2 \mathrm{~mm}$ and $0.9 \mathrm{~mm}$ outer diameter are fabricated. Often initial calibration conditions can not be maintained during the measurements. One such example is the microwave connection problems that may arise due to the probe movements. Therefore, there is a need to recalibrate the system after few measurements. To mitigate the calibration problem and increase the measurement repeatability the probes are fabricated with an integrated cable. The fabricated probe with $2.2 \mathrm{~mm}$ aperture diameter is shown in Fig. 3 (b). The probe is connected to Agilent Fieldfox Handheld RF and Microwave Analyzer N9923A which is used for S-parameter measurements. The analyzer was connected to a laptop computer and the dielectric property measurements were also recorded with the Agilent 85070 Dielectric property measurement software for comparison. Measurement set-up is shown in Fig. 3 (a).

Proper functioning of the fabricated probes are first tested with the known materials. Conventional calibration procedure; namely, open, short, deionized water is followed before the measurements. Methanol is used as the known material. Comparison of permittivity and conduc-

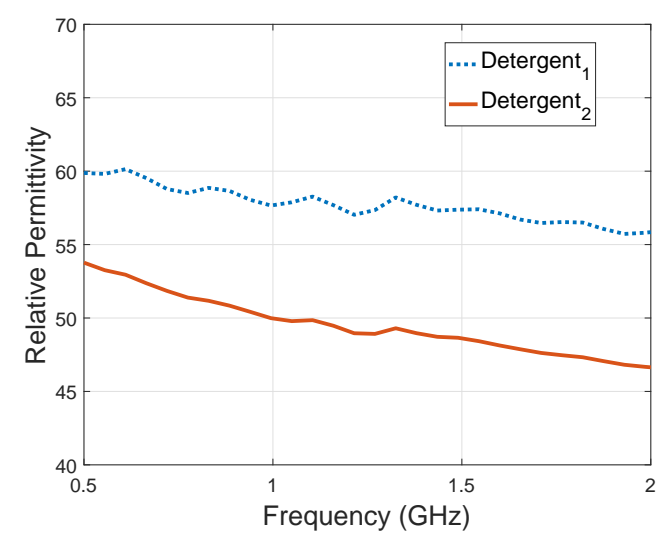

(a)

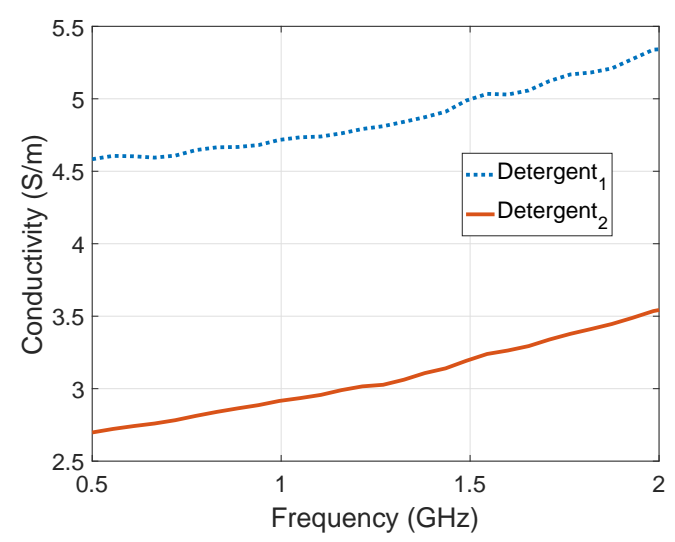

(b)

Figure 2: Measured dielectric properties of the detergents (a) Permittivity comparison of the detergent ${ }_{1}$ and detergent $_{2}$ (b) Conductivity comparison of the detergent ${ }_{1}$ and detergent . $_{2}$ 


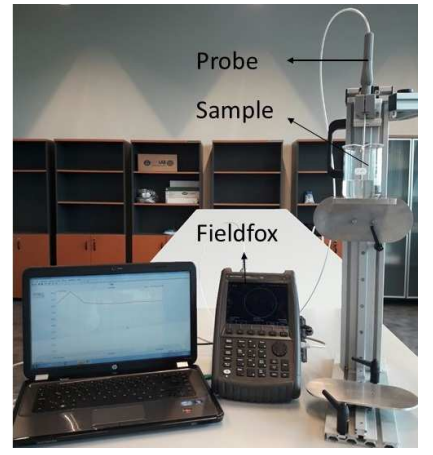

(a)

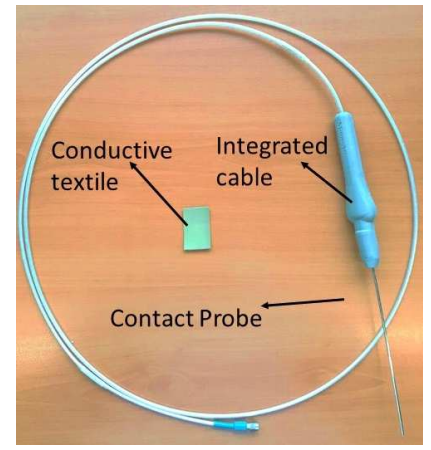

(b)

Figure 3: Fabricated contact probe with $2.2 \mathrm{~mm}$ outer diameter(a) measurement set-up (b) Contact probe integrated with the cable.

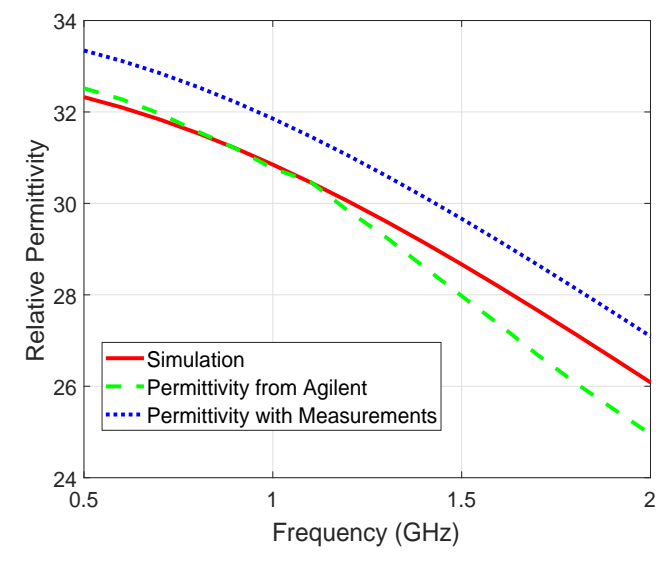

(a)

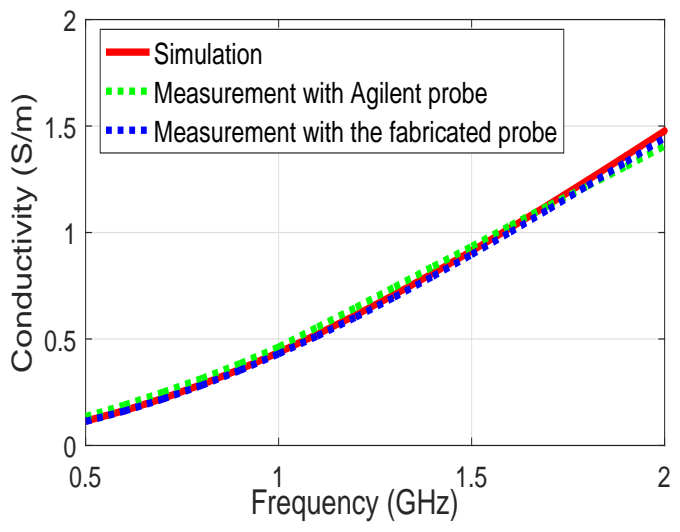

(b)

Figure 4: Methanol dielectric property measurement and simulation comparisons between $500 \mathrm{MHz}$ to 2 $\mathrm{GHz}$ for contact probe with $2.2 \mathrm{~mm}$ aperture diameter (a) relative permittivity $\left(\epsilon_{r}\right)$ (b) conductivity $(\sigma /$ $\mathrm{S} / \mathrm{m})$.

tivity results for probe with $2.2 \mathrm{~mm}$ aperture diameter is shown in Fig. 4(a) and Fig. 4(b), respectively. Simulation results represents the retrieved dielectric properties obtained from simulated S-parameter results of the probe with $2.2 \mathrm{~mm}$ in aperture diameter. Two measurements are performed, as a reference measurement the dielectric properties of methanol is measured with the commercial Agilent slim-form contact probe and the dielectric property measurement is also performed with the Agilent 85070 dielectric property measurement software. Also, S-parameter measurement is performed with the fabricated 2.2 aperture diameter probe and dielectric property retrieval is performed with the in-house retrieval algorithm. From the measurement and simulation results it can be seen that the measurement and simulation results agree well for the permittivity and conductivity results. The discrepancy between the commercial probe and fabricated probe is expected to increase at higher frequencies. The probe is not expected to perform well over 10 GHz. Although the frequency limitation can be improved by choosing an appropriate cable, it is expected that measurement between $500 \mathrm{MHz}$ to $6 \mathrm{GHz}$ should be adequate for most of the envisioned diagnostics applications of the probe.

Similarly performance of the fabricated probe with an aperture diameter of $0.9 \mathrm{~mm}$ is evaluated with the known material; that is, methanol measurements. First the performance of the probe is evaluated in the simulation environment where the probe was simulated in CST with the tip immersed in the box with methanol dielectric properties. Measurements are performed with the measurement set-up described in Section 2.3 and the Agilent 85070E software software is used for dielectric property retrieval. Next, dielectric properties obtained from simulations and measurements are compared with the dielectric property measurements collected with the probe with 2.2 $\mathrm{mm}$ aperture diameter. Comparison of permittivity and conductivity results for probe with $0.9 \mathrm{~mm}$ 


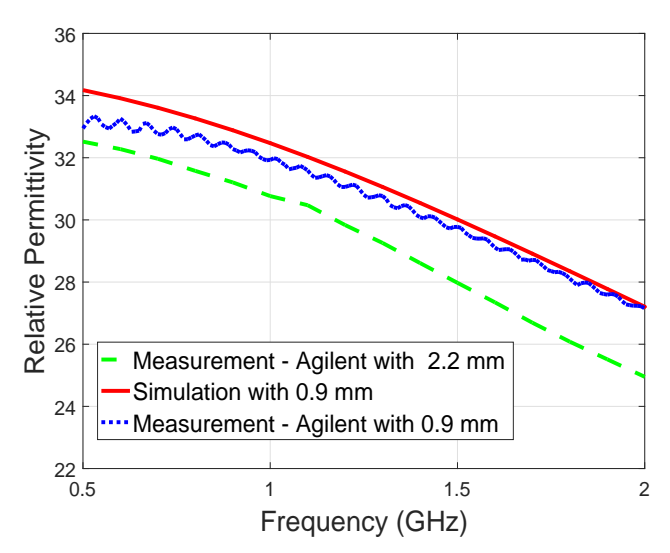

(a)

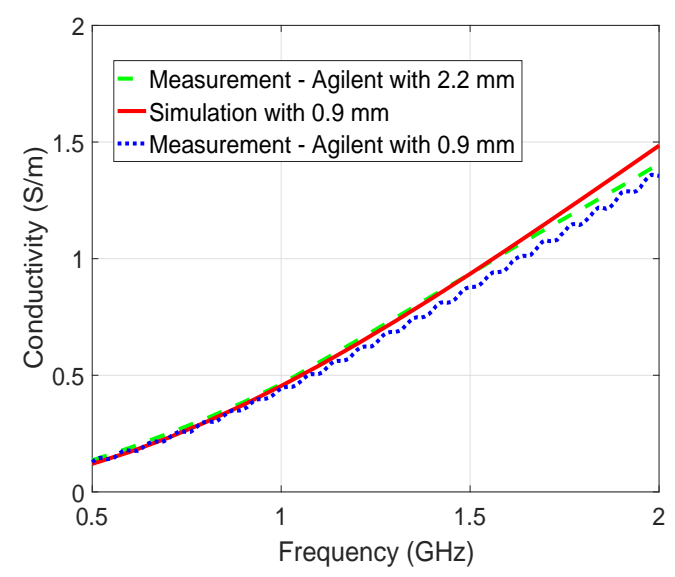

(b)

Figure 5: Methanol dielectric property measurement and simulation comparisons between $500 \mathrm{MHz}$ to 2 $\mathrm{GHz}$ for contact probe with $0.9 \mathrm{~mm}$ aperture diameter (a) relative permittivity $\left(\epsilon_{r}\right)($ b) conductivity $(\sigma /$ $\mathrm{S} / \mathrm{m})$.

aperture diameter is shown in Fig. 5(a) and Fig. 5(b), respectively. Permittivity obtained from the simulation and measurement of the methanol with $0.9 \mathrm{~mm}$ probe agrees well. The permittivity obtained from the measurement with $2.2 \mathrm{~mm}$ probe is $1-2$ units lower than the $0.9 \mathrm{~mm}$ probe. The results for methanol measurements agrees well. However, the discrepancy between the $0.9 \mathrm{~mm}$ and $2.2 \mathrm{~mm}$ probe could be mitigated by using the in-house dielectric property retrieval algorithm.

After the verification of proper operation of the probes with methanol, dielectric properties of the characterized phantom materials are measured with fabricated $2.2 \mathrm{~mm}$ and $0.9 \mathrm{~mm}$ probes. Measured permittivity and conductivity of the fat mimicking material is shown in Fig. 6(a) and Fig. 6(b), respectively. Measured dielectric property values of that fat mimicking phantom material agrees well. Relative permittivity and conductivity of breast fat is given as 5.5 and $0.04 \mathrm{~S} / \mathrm{m}$ at 500 $\mathrm{MHz}$, respectively [3]. Measured relative permittivity and conductivity of the phantoms are slightly higher than the values reported in the literature. Dielectric properties of the wet-skin mimicking material is also measured with the fabricated probes. Measured permittivity and conductivity of the wet-skin mimicking material is shown in Fig. 7(a) and Fig. 7(b), respectively. Since both the relative permittivity and conductivity of the wet-skin mimicking phantom is higher than the fat mimicking material the difference between the measured results increases by 5 units for relative permittivity and $0.1 \mathrm{~S} / \mathrm{m}$ for conductivity. Dielectric properties of the malignant breast tumour mimicking material is also measured with both of the fabricated probes. Measured permittivity and conductivity of the breast tumour mimicking material is shown in Fig. 8(a) and Fig. 8(b), respectively. Similar to the wet-skin mimicking material phantom relative permittivity measurement discrepancy between two probes is 4 units.' The permittivity measured with the $0 / 9 \mathrm{~mm}$ probe is consistently gives higher values for materials with high permittivities. This could either be due to the sensing depth difference or due to the dielectric property retrieval algorithm. Note that the main goal of this work is to utilize the open=ended contact probe technique as a diagnostic tool. Therefore, measurement should be repeatable and reliable. Thus, further work is needed to evaluate the repeatability and reliability of the probes. For the follow-up of this work, the Sparameter responses will be further investigated and other probes will be fabricated to improve the performance of open-ended contact probes.

\section{CONCLUSION}

In this work, open-ended contact probes with different apertures are designed with an integrated RF cable. Contact probe with $2.2 \mathrm{~mm}$ and $0.9 \mathrm{~mm}$ aperture diameters are fabricated and tested with known materials. S parameter measurements were collected and dielectric properties of the MUT is retrieved with an in-house algorithm. The measurements were also compared with measurements taken with a commercial probe. A good agreement is obtained between the fabricated and commercial probe measurements. The probes are then tested with characterized gel-like phantom materials. Dielectric property measurement results for the phantom materials slightly varied 


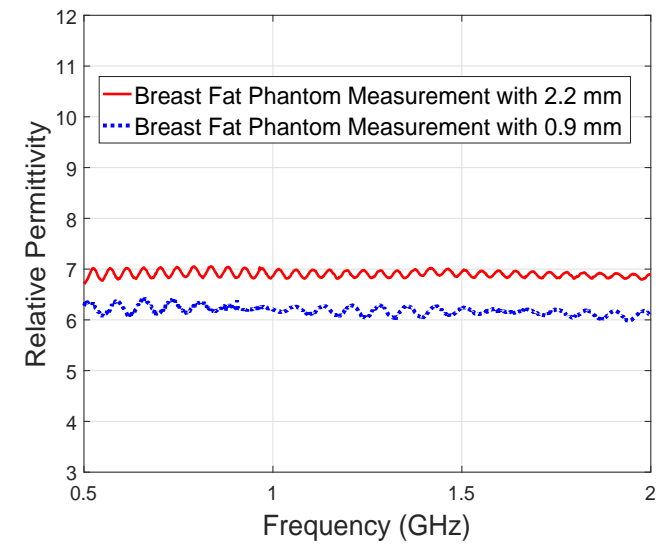

(a)

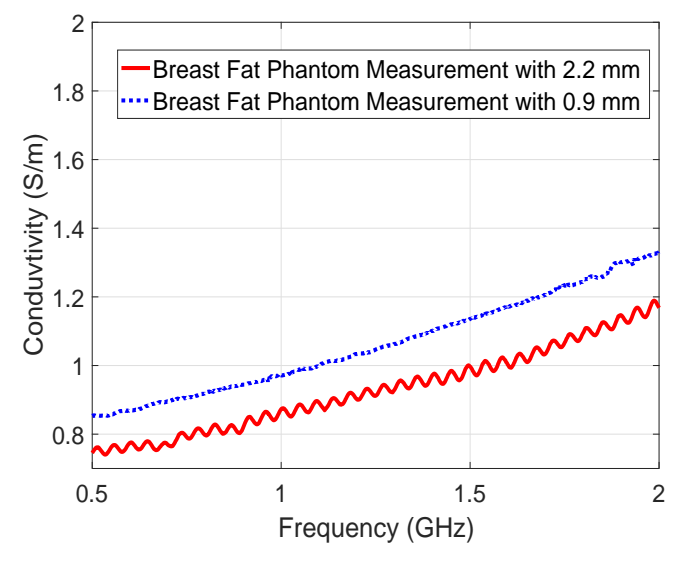

(b)

Figure 6: Fat mimicking phantom dielectric property measurement performed via fabricated probes with 2.2 $\mathrm{mm}$ and $0.9 \mathrm{~mm}$ aperture diameters (a) relative permittivity $\left(\epsilon_{r}\right)(\mathrm{b})$ conductivity $(\sigma / \mathrm{S} / \mathrm{m})$.

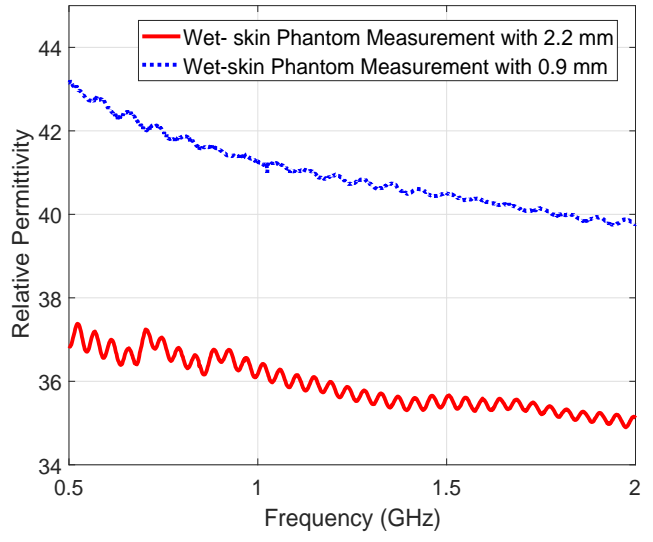

(a)

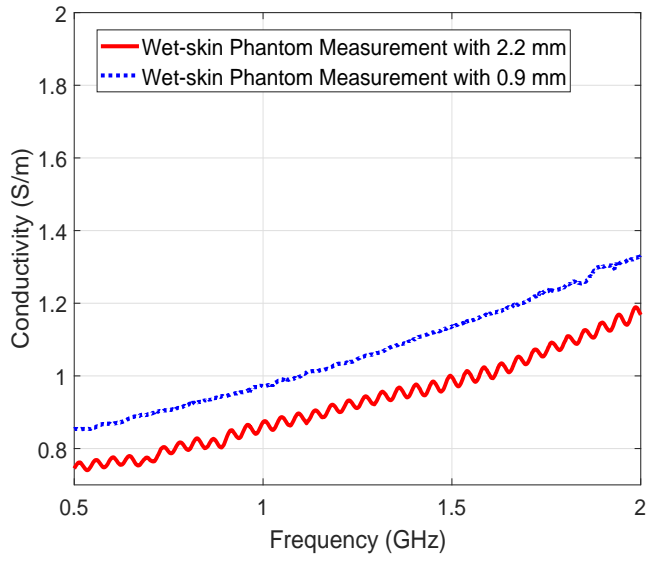

(b)

Figure 7: Wet-skin mimicking phantom dielectric property measurement performed via fabricated probes with $2.2 \mathrm{~mm}$ and $0.9 \mathrm{~mm}$ aperture diameters (a) relative permittivity $\left(\epsilon_{r}\right)(\mathrm{b})$ conductivity $(\sigma / \mathrm{S} / \mathrm{m})$.

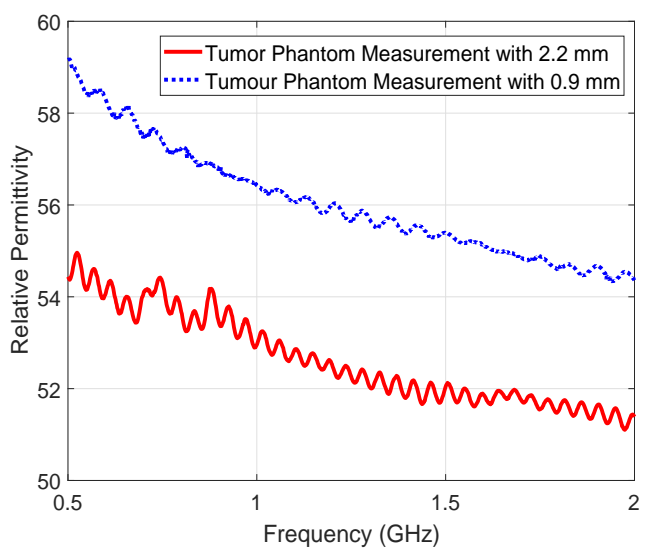

(a)

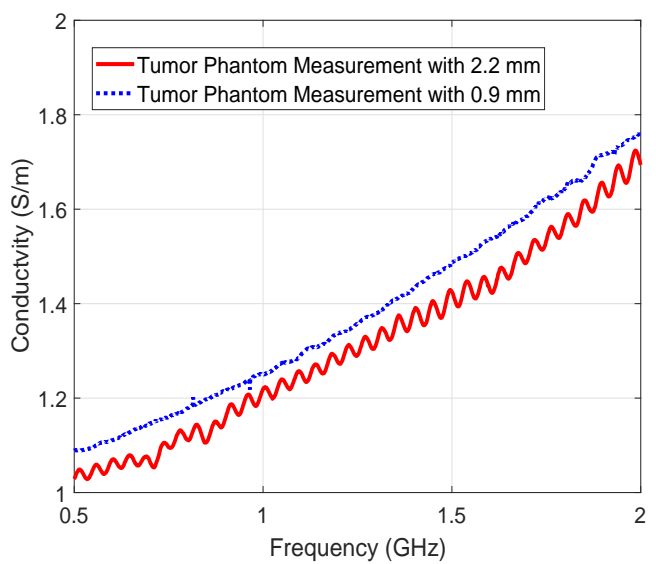

(b)

Figure 8: Malignant tumour mimicking phantom dielectric property measurement performed via fabricated probes with $2.2 \mathrm{~mm}$ and $0.9 \mathrm{~mm}$ aperture diameters (a) relative permittivity $\left(\epsilon_{r}\right)(\mathrm{b})$ conductivity $(\sigma / \mathrm{S} / \mathrm{m})$. 
for probes with different apertures. The discrepancy of dielectric property measurements between two probes is higher for phantoms with high dielectric properties. This variation is expected to be minimized through utilization of inhouse dielectric property retrieval algorithm.

\section{ACKNOWLEDGMENT}

This project has received funding from the European Union's Horizon 2020 research and innovation programme under the Marie Sklodowska-Curie grant agreement No 750346.

\section{REFERENCES}

1. Komarov, Sergey A., et al. "Open-ended coaxial probe technique for dielectric spectroscopy of artificially grown sea ice." IEEE Transactions on Geoscience and Remote Sensing 54.8 (2016): 4941-4951.

2. Gabriel, Camelia, Gabriel, Sami and Corthout, Y. E. "The dielectric properties of biological tissues: I. Literature survey." Physics in medicine \& biology 41.11 (1996): 2231.

3. Gabriel, Sami, Lau, R. W., and Gabriel, Camelia, "The dielectric properties of biological tissues: II. Measurements in the frequency range $10 \mathrm{~Hz}$ to $20 \mathrm{GHz}, "$ Physics in medicine $\&$ biology, Vol. 41, No. 11, 2251, 1996.

4. Gabriel, Sami, Lau, R. W. and Gabriel, Camelia." The dielectric properties of biological tissues: III. Parametric models for the dielectric spectrum of tissues." Physics in medicine 83 biology 41.11 (1996): 2271.

5. Lazebnik, M., Popovic, D., McCartney, L., Watkins, C. B., Lindstrom, M. J., Harter, J., ... \& Temple, W., "A large-scale study of the ultrawideband microwave dielectric properties of normal, benign and malignant breast tissues obtained from cancer surgerie," Physics in medicine $\&$ biology, Vol. 52, No. 20, 6093, 2007.

6. Lazebnik, M., McCartney, L., Popovic, D., Watkins, C. B., Lindstrom, M. J., Harter, J., ... \& Hagness, S. C. "A large-scale study of the ultrawideband microwave dielectric properties of normal breast tissue obtained from reduction surgeries," Physics in medicine $\&$ biology, Vol. 52, No. 10, 2637, 2007.

7. O'Rourke, A. P., Lazebnik, M., Bertram, J. M., Converse, M. C., Hagness, S. C., Webster, J. G., \& Mahvi, D. M., "Dielectric properties of human normal, malignant and cirrhotic liver tissue: in vivo and ex vivo measurements from 0.5 to $20 \mathrm{GHz}$ using a precision open-ended coaxial probe," Physics in medicine \& biology, Vol. 52, No. 15, 4707, 2007.

8. Yilmaz, T., Kılıç, M. A., Erdoğan, M., Çayören, M., Tunaoğlu, D., Kurtoğlu, İ., ... \& Cancan, G., "Machine learning aided diagnosis of hepatic malignancies through in vivo dielectric measurements with microwaves," Physics in medicine \& biology, Vol. 61, No. 13, 5089, 2016.

9. Anderson, W., Microwave biopsy probe,U.S. Patent Application No 10/961, 812, 2007.

10. Yilmaz, T., Foster, R., \& Hao, Y., "Broadband tissue mimicking phantoms and a patch resonator for evaluating noninvasive monitoring of blood glucose levels," IEEE Transactions on Antennas and Propagation, Vol. 62, No. 6, 3064-3075, 2014.

11. Yilmaz, Tuba, Robert Foster, and Yang Hao. "Towards accurate dielectric property retrieval of biological tissues for blood glucose monitoring." IEEE transactions on Microwave Theory and Techniques 62.12 (2014): 3193-3204.

12. Yilmaz, Tuba, Tutku Karacolak, and Erdem Topsakal. "Characterization and testing of a skin mimicking material for implantable antennas operating at ISM band $(2.4 \mathrm{GHz}-2.48 \mathrm{GHz}) . "$ IEEE Antennas and Wireless Propagation Letters 7 (2008): 418-420.

13. Keysight N1501A., "Dielectric Probe Kit $10 \mathrm{MHz}$ to $50 \mathrm{GHz}$," in https://literature.cdn.keysight.com/litweb/pdf/5992-0264EN.pdf?id=2605692, accessed May 2018.

14. Lazebnik, Mariya, Madsen, Ernest L, Frank, Gary R and Hagness, Susan C. "Tissue-mimicking phantom materials for narrowband and ultrawideband microwave applications." Physics in medicine \& biology 50.18 (2005): 4245.

15. Kim, Jaehoon, and Yahya Rahmat-Samii. "Implanted antennas inside a human body: Simulations, designs, and characterizations." IEEE Transactions on microwave theory and techniques 52.8 (2004): 1934-1943. 